\title{
Osteopathic manipulative medicine in the management of headaches associated with postconcussion syndrome
}

https://doi.org/10.1515/jom-2020-0035

Received February 24, 2020; accepted March 8, 2021;

published online April 9, 2021

\section{Abstract}

Context: Previous studies have demonstrated the effectiveness of osteopathic manipulative treatment (OMT) for various headache types, with limited evidence of its use for headaches related to mild traumatic brain injury (MTBI). No studies prior studies were found regarding OMT for headaches in patients with postconcussion syndrome (PCS), defined as symptom persistence for longer than 3 months after MTBI.

Objectives: To evaluate OMT for headaches in patients with PCS.

Methods: A controlled pilot study was conducted of patients with PCS who presented to an outpatient interdisciplinary rehabilitation clinic; patients with symptoms lasting longer than 3 months were enrolled and randomly assigned to an OMT treatment group or a control group. Primary outcome measures were immediate change in headache scores according to a Visual Analog Scale (VAS) and change in the six item Headache Impact Test (HIT-6) between baseline and follow up visits. The participants in the control group completed the HIT- 6 between baseline and follow up visits but did not receive OMT and did not complete the VAS. Mean immediate VAS score change for the treatment group and mean improvement in HIT-6 scores for both groups between baseline and follow up were analyzed for statistical significance.

*Corresponding author: Dmitry Esterov, DO, Department of Physical Medicine and Rehabilitation, Mayo Clinic, 200 First St SW, Rochester, MN 55905, USA, E-mail: esterov.dmitry@mayo.edu Alphonsa Thomas, DO, Department of Physical Medicine and Rehabilitation, Hackensack Meridian Health, Shore Rehabilitation Institute, Brick Township, NJ, USA

Kyle Weiss, DO, St. Luke's Spine and Pain Medicine Associates, Easton, PA, USA
Results: A total of 26 patients were included in this study: $13(50 \%)$ in the treatment group and $13(50 \%)$ in the control group. Six patients (23.1\%), three from each group, did not complete the study, so 10 subjects in each group were included in the final analysis. Statistically significant improvement in VAS scores was seen immediately after OMT in the treatment group (mean change, 2.1; $\mathrm{p}=0.002$ ). Mean HIT-6 scores showed improvement in the treatment group compared with the control group, although the change was not statistically significant $(p=0.15)$ from baseline to follow up visit. No adverse effects from treatments were noted.

Conclusions: Patients with headaches secondary to PCS showed immediate benefit in headache pain intensity after OMT. However, no sustained benefit was found on the follow up visit compared with the control group.

Keywords: concussion; neurorehabilitation; physical medicine and rehabilitation; postconcussion syndrome; posttraumatic headache; osteopathic manipulative treatment; rehabilitation; traumatic brain injury.

Mild traumatic brain injury (MTBI) is a disruption in brain function caused by a traumatic biomechanical force to the head, associated with loss of consciousness for less than 30 minutes, posttraumatic amnesia lasting less than 24 hours, and an initial Glasgow Coma Scale of 13-15 [1]. Although the majority of patients recover within a few weeks after MTBI, an estimated 5-43\% have persistent symptoms, which include cognitive difficulties, visual dysfunction, sleep disturbance, emotional changes, fatigue, tinnitus, headaches, dizziness, and balance impairment $[2,3]$. These symptoms often become characterized as postconcussion syndrome (PCS) when they last longer than 3 months [3]. They are associated with a lower quality of life and increased disability [4].

Headache is the most common physical condition reported after traumatic brain injury (TBI) and occurs more frequently in patients with MTBI than those with more severe injuries [3, 5]. Posttraumatic headache (PTH), as defined by the International Classification of Headache 
Disorders Third Edition [6], is a secondary headache disorder attributed to trauma to the head, neck, or both in temporal relationship to the injury. The prevalence of PTH is estimated between 30 and $90 \% ; 18-22 \%$ of patients have symptoms for longer than 1 year despite a wide variability of existing treatment approaches [5].

Given that symptoms after MTBI often persist despite ongoing treatment [3], continued study of therapeutic options is important to assist with recovery. Osteopathic manipulative treatment (OMT) has been found to be effective in improving neck pain and various types of headache [7-9]. There is growing evidence that untreated pathology of the cervical spine secondary to the whiplash injury associated with MTBI is an important contributor to persistent PCS symptoms, including chronic PTH [10, 11]. The use of targeted OMT to restore normal muscle tone and range of motion, particularly to the cervical spine, can thus theoretically assist in the management of PTH associated with whiplash injury and traumatic biomechanical forces to the head [7, 12-14].

There is limited evidence showing benefit of OMT in improving acute symptoms after MTBI [12, 15-19]. Evidence is even scarcer regarding the utility of OMT for chronic symptoms associated with PCS. Channell et al. [20] described the benefit of OMT with multidisciplinary treatment of a patient with chronic PTH, defined as headache occurring 3 months after MTBI. To our knowledge, that case report [20] is the only study of the effect of OMT in a patient with PTH.

The primary aim of this pilot study was to assess the efficacy of OMT in the treatment of chronic PTH associated with PCS. We sought to assess the safety and efficacy of OMT in providing an immediate benefit for patients with chronic PTH, and to determine whether sustained improvement would be seen at a predetermined follow up visit when compared with results from a control group who did not receive OMT. We hypothesized that OMT would be safe and have a benefit both immediately and on a follow up visit at approximately 1 month after treatment.

\section{Methods}

This prospective pilot study was approved by the Institutional Review Board the JFK Johnson Rehabilitation Institute and was registered post hoc at ClinicalTrials.gov (no. NCT04732338).

All potential participants were patients seeking treatment for MTBI and were initially evaluated by a board certified brain rehabilitation physiatrist not otherwise affiliated with the study in an outpatient interdisciplinary rehabilitation clinic, who confirmed the diagnosis of MTBI and initial date of injury. That physician did not perform a structural osteopathic examination and did not provide OMT. After initial evaluation, all participants deemed potentially eligible were evaluated by one of three authors (D.E., A.T., or K.W.) who are board certified osteopathic physicians specializing in physical medicine and rehabilitation; they reviewed eligibility criteria and oversaw the informed consent process.

Inclusion criteria were a diagnosis of MTBI [1], age 18 years or older, injury occurring 3 months or more prior to presentation, and headache as a primary symptomatic concern. Exclusion criteria were a history of moderate to severe TBI, documented intracranial injury, chronic headache or migraine headache before the injury, treatment by a headache specialist at the time of injury, or receipt of intravenous medication infusion headache at the time of initial treatment. Patients with a premorbid psychological history or history of chronic pain, attention deficit hyperactivity disorder (ADHD), or prior MTBI were not excluded, given the high association between concomitant chronic TBI symptoms and these conditions [4, 21].

Between January 1, 2018, and March 31, 2019, participants were enrolled and assigned to an OMT treatment group or control group without OMT. Researchers alternated assigning patients between treatment and control groups after participants signed informed consent. The number of patients screened for eligibility was not documented.

Evaluation of patients in the treatment group was performed by one of three treatment providers (D.E., A.T., or K.W.), and consisted of a musculoskeletal examination and an osteopathic structural examination. A structural examination was not performed for participants in the control group. Participants and physicians providing treatment were not blinded. All participants received conventional care for MTBI (i.e., rehabilitation therapy with physical, occupational, or speech therapy, education regarding symptom management, over the counter medications), did not seek treatment from additional providers specifically for headache between follow up visits, and did not receive additional prescribed medications for headache.

Prior to the beginning of the study and initiation of treatment, OMT approaches were determined by the treatment providers (D.E., A.T., and K.W.) [22]. Muscle energy, myofascial release, counterstain, and suboccipital release were chosen as the treatments, as they are among the most commonly used techniques [23], with least risk of adverse effects [24], that were able to be performed with the most consistency (according to mutual consensus of the providers) between all treating providers in this study independent of level of experience. OMT was performed in a supine position, with treatments targeted to the cervicothoracic region. There was no particular sequence to treatment, though all four techniques were performed for every participant in the treatment group.

Primary outcome measures were headache scores on a Visual Analog Scale (VAS) and scores on the six item Headache Impact Test (HIT-6). The VAS is a simple and frequently used measure for assessment of pain intensity, shown to be reliable and valid in various rehabilitation interventions [25]. The VAS was scored on a 0 to 10 scale, with 0 signifying no pain and 10 signifying the worst possible headache pain. The HIT- 6 is a measure of headache severity and provides information regarding the effect of headache on other domains related to functional participation [26]. It is valid and reliable in monitoring of treatment of headache in clinical and research settings $[26,27]$. The HIT-6 has six questions and a maximum score of 78. Higher HIT- 6 scores indicate increased headache severity and greater functional limitations [27].

Participants in the treatment group completed a HIT-6 test and quantified their current headache pain with a VAS before OMT, while participants in the control group completed a HIT- 6 only. Participants 
in the treatment group were asked to complete a VAS immediately after treatment, and any adverse effects were recorded. During the next follow up visit with the referring provider between 4 and 6 weeks posttreatment, treatment and control participants completed a subsequent HIT-6. HIT-6 was chosen as the follow up outcome measure rather than to VAS because the HIT- 6 is a measure of the symptoms over the prior 4 weeks [27] instead of a single time point; thus, HIT-6 was thought to better reflect an average change of headache symptoms over time.

\section{Data analysis}

JMP version 14.1 (SAS Institute, Inc.) was used for statistical analysis. Descriptive statistics (mean, median, standard deviation [SD]) and graphing (box plots, bar charts) methods were used. For the treatment group, a paired Wilcoxon signed rank test was performed to assess changes in VAS data from pretreatment to posttreatment. With 10 patients, there was $89 \%$ power to detect a change of 2.0 in the VAS scores from pretreatment to posttreatment, assuming a 2 sided significance level of $5 \%$ and a standard deviation of 2.0 (effect size $=1$ ). The Wilcoxon rank sum test for continuous (e.g., age) and discrete variables (e.g., change in HIT-6 scores, time from injury to visit [months]) and the Pearson $\chi^{2}$ test for categorical variables (e.g., mechanism of injury, history of prior MTBI) were performed for comparison of the treatment and control groups. With 20 patients (10 per group), we had $80 \%$ power to detect a difference in the means of 10 between the groups in HIT-6, assuming a standard deviation of 8.0, and a two sided significance level of $5 \%$. Two sided $\mathrm{p}<0.05$ was considered statistically significant.

\section{Results}

A total of 26 patients were included in this study: 13 (50\%) in the treatment group and $13(50 \%)$ in the control group. Six patients (23.1\%), three patients from each group, did not complete the study, so 10 subjects in each group were included in the final analysis.
On structural examination, all patients in the treatment group were found to have somatic dysfunction.

Table 1 summarizes baseline characteristics between the treatment and control groups. No statistically significant pretreatment demographic differences were observed between groups. The largest difference between groups was the time from injury to evaluation, with a mean of 12 months until evaluation in the treatment group (SD, 8 months) and 29 months until evaluation in the control group (SD, 27 months; $\mathrm{p}=0.08$ ).

Figure 1 shows the change in VAS score in participants who received OMT. The mean change between baseline VAS scores (mean, 5.6 [SD, 1.7]) and immediate posttreatment scores (mean, 3.5 [SD, 2.1]) was 2.1 [SD, 1.8], which was statistically significant $(\mathrm{p}=0.002)$. There were no adverse effects noted with treatment.

Table 2 outlines changes in HIT- 6 scores between baseline and follow up visits. The treatment group had a

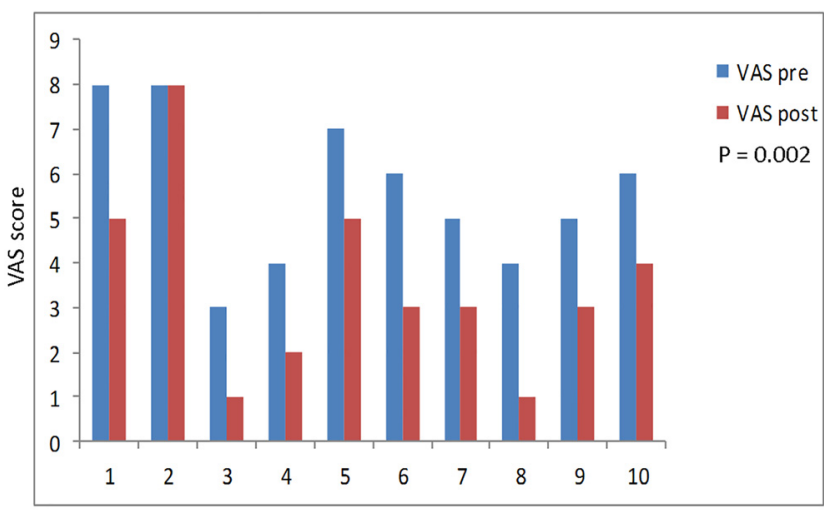

Participant

Figure 1: Change in Visual Analog Scale (VAS) scores immediately after osteopathic manipulative treatment in the treatment group.

Table 1: Baseline characteristics of study participants.

\begin{tabular}{|c|c|c|c|}
\hline Characteristic & Treatment group $(n=10)$ & Control group $(n=10)$ & p-value ${ }^{a}$ \\
\hline Age at evaluation, years, mean (SD; range) & $45(15 ; 20-71)$ & $42(17 ; 18-64)$ & $>0.99$ \\
\hline \multicolumn{4}{|l|}{ Sex } \\
\hline Female & 7 & 6 & 0.64 \\
\hline Male & 3 & 4 & \\
\hline History of prior MTBI & 3 & 4 & 0.64 \\
\hline History of depression & 4 & 3 & 0.64 \\
\hline Time to evaluation since MTBI, months, mean (SD; range) & $12(8 ; 3-23)$ & $29(27 ; 4-88)$ & 0.08 \\
\hline Mechanism of injury & & & 0.53 \\
\hline MVC & 8 & 6 & \\
\hline Fall & 1 & 3 & \\
\hline Sports & 1 & 1 & \\
\hline
\end{tabular}

MTBI, mild traumatic brain injury; MVC, motor vehicle crash. ${ }^{a}$ Wilcoxon rank sum test was performed for continuous variables (e.g., age, time from injury to visit [mo]); Pearson $\chi^{2}$ test for categorical variables (e.g., mechanism of injury, history of prior MTBI). 


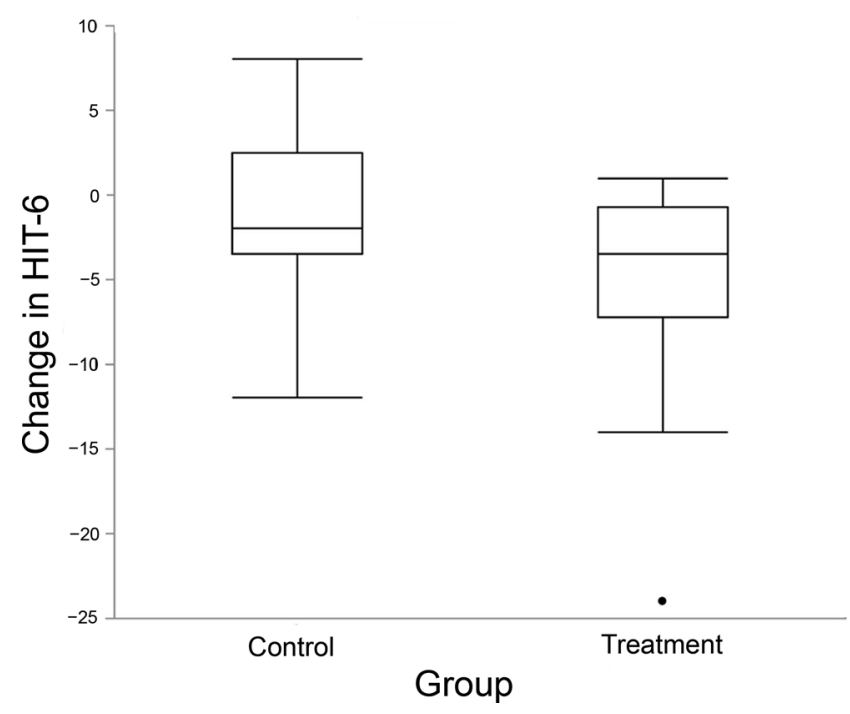

Figure 2: Change in the six item Headache Impact Test (HIT-6) scores between initial (baseline) and 4 to 6 week follow up visit in both treatment and control groups. A negative change represents a decreased HIT-6 score on follow up visit, indicative of an improvement in headache symptoms.

mean score improvement of a 5.7 points improvement, while the control group had a mean score improvement of 1.1 points (Figure 2); no statistically significant difference was seen between HIT-6 scores from baseline to follow up visit in either group $(\mathrm{p}=0.15)$.

\section{Discussion}

This was a prospective pilot study analyzing the use of OMT for chronic headache associated with PCS. No adverse effects or worsening of symptoms were found after OMT, and a significant improvement in headache severity immediately after OMT was found. While there was a difference in mean HIT- 6 scores during 4 to 6 week follow up visits showing greater improvement in the treatment

Table 2: Group comparison of change in HIT-6 scores.

\begin{tabular}{llrrr}
\hline HIT-6 variable & Group & $\begin{array}{r}\text { Score, } \\
\text { mean (SD) }\end{array}$ & $\begin{array}{r}\text { Score, } \\
\text { median (Q1, Q3) }\end{array}$ & \\
\hline Baseline & Treatment & $63.8(7.2)$ & $63.0(57.8,70.0)$ & 0.27 \\
& Control & $56.5(14.0)$ & $62.0(49.5,65.5)$ & \\
Follow up & Treatment & $58.1(9.6)$ & $60.0(55.0,65.0)$ & 0.88 \\
& Control & $55.4(14.4)$ & $60.5(49.5,64.3)$ & \\
Change & Treatment & $5.7(7.7)$ & $3.5(0.8,7.3)$ & 0.15 \\
& Control & $1.1(5.5)$ & $2.0(-2.5,3.5)$ & \\
\hline
\end{tabular}

HIT-6, six item Headache Impact Test; SD, standard deviation; Q1, first quartile; Q3, third quarter. ${ }^{\text {} W i l c o x o n ~ r a n k ~ s u m ~ t e s t . ~}$ group, no statistically significant benefit was found. These overall findings are suggestive that immediate improvement in headache pain can occur in patients with PCS after OMT; however, results are inconclusive to demonstrate a sustained effect on follow up.

There is increasing evidence that untreated musculoskeletal pathology of the cervical spine secondary to whiplash associated with injury is an important contributor to chronic symptoms after MTBI [10, 11]. Dysfunctions in the cervical zygapophysial joints from injury due to traumatic biomechanical forces can result in hyperexcitability of nociceptive response in the central nervous system, contributing to headache $[10,28]$. In addition, afferent nerve fibers from $\mathrm{C} 2$ and $\mathrm{C} 3$ dorsal root ganglia terminate on the same second order neurons, as do those arising from trigeminal sensory nucleus. This convergence, known as the trigeminocervical nucleus, provides a mechanism for headache caused by dysfunction in the upper cervical structures [11]. The improvement in VAS scores immediately after OMT in this study suggests that ongoing musculoskeletal pathology of the cervical spine may contribute to chronic PTH, and the use of techniques targeted to restore normalcy to the cervical spine may improve signaling from cervical mechanoreceptors and nociceptors [12], thus assisting in the management of PTH.

There are additional mechanisms that can contribute to PCS, including altered cerebral blood flow, labyrinthitis, autonomic nervous system changes, and cranial dysfunction [3, 10, 11, 15]. In addition, PCS symptoms have been reported in healthy people who did not sustain MTBI and in those with depression, chronic pain, ADHD, and posttraumatic stress disorder [21]. Various behavioral and psychological factors may contribute to persistent symptoms in this sample, and may contribute to a modest change in VAS scores overall [29]. The HIT-6 measures not only headache severity, but also the effect of headache on social, occupational, and cognitive functioning [26]. While a history of depression was found in four participants in the treatment group and three participants in the control group, other behavioral and psychological factors were not characterized with certainty. In this context, OMT should be used as part of an overall management process that includes consideration of these factors and elimination of other differential diagnoses. In addition, future studies could consider a subgroup analysis of treatment response in individuals with PCS and comorbid history of depression.

Because of the complex pathophysiology of PCS, patients with chronic symptoms may be more effectively treated with OMT as an adjunct to an individualized multidisciplinary approach [29, 30]. Ellis et al. [31] 
described an approach to identifying and treating PCS on the basis of identifying symptom subtypes. A multimodal impairment based approach has been effective in the improvement of overall symptoms [30, 32]. In a case report of a patient with chronic PTH, Channell et al. [20] reported improvement with multidisciplinary treatment that included OMT and psychological evaluation, pharmacologic interventions, and physical therapy. While our pilot study did not test a multidisciplinary intervention, future research could consider incorporating OMT as part of multimodal treatment.

\section{Limitations}

This pilot study had several limitations. The sample size in this study limited the power to assess for statistical significance of HIT- 6 scores between the treatment and control groups. Sample sizes of 35 in the treatment group and 35 in the control group would have achieved $80.8 \%$ power to reject the null hypothesis with a population mean difference detected in the change in HIT- 6 scores between treatment and control groups on follow up. Accounting for $20 \%$ dropout rate, sample sizes would include approximately 44 patients in treatment and 44 patients in the control group [33].

The OMT protocol in this study did not include cranial manipulation, Fulford Dural Release, or venous sinus drainage, which may improve symptoms further by targeting other mechanisms associated with PCS $[15,16,20,34$, 35]. Treating physicians were not blinded and only one treatment was performed, increasing variability of response and limiting understanding of the long term impact of OMT on PCS. Neither structural and musculoskeletal exams nor the treatment protocol were standardized, structural examination was not performed on the control group, and data on structural exams for treatment group were not recorded. A structural exam during follow up in treatment group was not performed. In addition, data acquisition on all comorbidities of participants was incomplete. Participants underwent some unmeasured treatments (conventional care including rehabilitation therapy, education, over the counter medications), making it difficult to isolate the effect of OMT in the overall recovery.

Future studies should address these limitations and consider the use of sham OMT treatment to control for the effects of nonspecific touch. The potential treatment effect of therapeutic touch in the treatment group cannot be determined from our results. The improvement represented by VAS scores should be interpreted in context of these limitations.

\section{Conclusions}

This pilot study, which analyzed the use of OMT for chronic headache associated with PCS, showed immediate benefit from OMT in improving headache severity in a sample with PCS. No additional benefit was observed compared with the control group at the 4 to 6 week follow up visit. Future studies should consider the use of a larger sample size and a prospectively determined, standardized treatment protocol focused on functional outcomes, in the assessment of the effect of OMT for patients with PCS.

Acknowledgments: The authors thank Ekua Gilbert Baffoe, $\mathrm{MD}$, for assistance with patient recruitment and data collection.

Research funding: The data analysis of this research was supported by Grant Number UL1 TR002377 from the National Center for Advancing Translational Sciences (NCATS). The contents of the study are solely the responsibility of the authors and do not necessarily represent the official views of the National Institutes of Health. The funder did not have any involvement in the decision to submit the manuscript for publication.

Author contributions: All authors provided substantial contributions to conception and design, acquisition of data, or analysis and interpretation of data; all authors drafted the article or revised it critically for important intellectual content; all authors gave final approval of the version of the article to be published; and all authors agree to be accountable for all aspects of the work in ensuring that questions related to the accuracy or integrity of any part of the work are appropriately investigated and resolved.

Competing interests: None reported.

Informed consent: All patients in this study provided informed consent prior to group assignment.

Ethical approval: This study was approved by Institutional Review Board at JFK Johnson Rehabilitation Institute (Edison, New Jersey). It was registered post hoc at ClinicalTrials.gov (no. NCT04732338).

\section{References}

1. Kay T, Harrington DE, Adams R, Anderson T, Berrol S, Cicerone K. Definition of mild traumatic brain injury: developed by the mild traumatic brain injury committee of the head injury interdisciplinary special interest group of the American Congress of Rehabilitation Medicine. J Head Trauma Rehabil 1993;8:86-7. 
2. Dikmen S, Machamer J, Temkin N. Mild traumatic brain injury: longitudinal study of cognition, functional status, and posttraumatic symptoms. J Neurotrauma 2017;34:1524-30.

3. Hiploylee C, Dufort PA, Davis HS, Wennberg RA, Tartaglia MC, Mikulis D, et al. Longitudinal study of postconcussion syndrome: not everyone recovers. J Neurotrauma 2017;34:1511-23.

4. Lange RT, Iverson GL, Rose A. Depression strongly influences postconcussion symptom reporting following mild traumatic brain injury. J Head Trauma Rehabil 2011;26:127-37.

5. Hoffman JM, Lucas S, Dikmen S, Braden CA, Brown AW, Brunner R, et al. Natural history of headache after traumatic brain injury. J Neurotrauma 2011;28:1719-25.

6. Tapia RN, Eapen BC, Cifu DX. Neurosensory disorders in mild traumatic brain injury. San Diego, California: Elselvier Inc; 2019.

7. Zwibel H, Leder A, Yao S, Finn C. Concussion evaluation and management: an osteopathic perspective. J Am Osteopath Assoc 2018;118:655-61.

8. Rolle G, Tremolizzo L, Somalvico F, Ferrarese C, Bressan LC. Pilot trial of osteopathic manipulative therapy for patients with frequent episodic tension-type headache. J Am Osteopath Assoc 2014;114:678-85.

9. Cerritelli F, Lacorte E, Ruffini N, Vanacore N. Osteopathy for primary headache patients: a systematic review. J Pain Res 2017; 10:601-11.

10. Morin M, Langevin P, Fait P. Cervical spine involvement in mild traumatic brain injury: a review. J Sports Med 2016;2016:1590161.

11. Marshall CM, Vernon H, Leddy JJ, Baldwin BA. The role of the cervical spine in post-concussion syndrome. Phys Sportsmed 2015;43:274-84.

12. Guernsey DT, III, Leder A, Yao S. Resolution of concussion symptoms after osteopathic manipulative treatment: a case report. J Am Osteopath Assoc 2016;116:e13-7.

13. Gallagher RM. Headache pain. J Am Osteopath Assoc 2005;105(4 Suppl):S7-11.

14. Schwerla F, Kaiser AK, Gietz R, Kastner R. Osteopathic treatment of patients with long-term sequelae of whiplash injury: effect on neck pain disability and quality of life. J Alternative Compl Med 2013;19:543-9.

15. Patel KG, Sabini RC. Safety of osteopathic cranial manipulative medicine as an adjunct to conventional postconcussion symptom management: a pilot study. J Am Osteopath Assoc 2018;118: 403-9.

16. Yao SC, Zwibel H, Angelo N, Leder A, Mancini J. Effectiveness of osteopathic manipulative medicine vs concussion education in treating student athletes with acute concussion symptoms. J Am Osteopath Assoc 2020;120:607-14.

17. Baltazar GA, Kolwitz C, Petrone P, Stright A, Joseph D. Osteopathic manipulative treatment relieves post-concussion symptoms in a case of polytrauma. Cureus 2020;12:e7317.

18. Chappell C, Dodge E, Dogbey G. Assessing the immediate effect of osteopathic manipulation on sports related concussion symptoms. Osteopath Fam Physician 2015;7:30-5.

19. Castillo I, Wolf K, Rakowsky A. Concussions and osteopathic manipulative treatment: an adolescent case presentation. J Am Osteopath Assoc 2016;116:178-81.
20. Channell MK, Mueller LL, Hahn R. Management of chronic posttraumatic headache: a multidisciplinary approach. J Am Osteopath Assoc 2009;109:509-13.

21. Iverson GL. Network analysis and precision rehabilitation for the post-concussion syndrome. Front Neurol 2019;10. https://doi. org/10.3389/fneur.2019.00489.

22. Association American Osteopathy. Foundations of osteopathic medicine. Baltimore, Maryland: Lippincott Williams \& Wilkins; 2010.

23. Ault B, Levy D. Osteopathic manipulative treatment use in the emergency department: a retrospective medical record review. J Am Osteopath Assoc 2015;115:132-7.

24. Degenhardt BF, Johnson JC, Brooks WJ, Norman L. Characterizing adverse events reported immediately after osteopathic manipulative treatment. J Am Osteopath Assoc 2018;118:141-9.

25. Crossley KM, Bennell KL, Cowan SM, Green S. Analysis of outcome measures for persons with patellofemoral pain: which are reliable and valid? Arch Phys Med Rehabil 2004;85: 815-22.

26. Yang M, Rendas-Baum R, Varon SF, Kosinski M. Validation of the headache impact test (HIT- $6^{\mathrm{TM}}$ ) across episodic and chronic migraine. Cephalalgia 2010;31:357-67.

27. Kosinski M, Bayliss MS, Bjorner JB, Ware JE Jr., Garber WH, Batenhorst $A$, et al. A six-item short-form survey for measuring headache impact: the HIT-6. Qual Life Res 2003;12:963-74.

28. Smith AD, Jull G, Schneider G, Frizzell B, Hooper RA, Sterling M. A comparison of physical and psychological features of responders and non-responders to cervical facet blocks in chronic whiplash. BMC Muscoskel Disord 2013;14:313. https://doi.org/10.1186/ 1471-2474-14-313.

29. Silverberg ND, laccarino MA, Panenka WJ, Iverson GL, McCulloch KL, Dams-O'Connor K, et al. Management of concussion and mild traumatic brain injury: a synthesis of practice guidelines. Arch Phys Med Rehabil 2019;101:382-93.

30. Grabowski P, Wilson J, Walker A, Enz D, Wang S. Multimodal impairment-based physical therapy for the treatment of patients with post-concussion syndrome: a retrospective analysis on safety and feasibility. Phys Ther Sport 2017;23:22-30.

31. Ellis MJ, Leddy JJ, Willer B. Physiological, vestibulo-ocular and cervicogenic post-concussion disorders: an evidence-based classification system with directions for treatment. Brain Inj 2015; 29:238-48.

32. Hugentobler JA, Vegh M, Janiszewski B, Quatman-Yates C. Physical therapy intervention strategies for patients with prolonged mild TBI symptoms: a case series. Int J Sports Phys Ther 2015;10:676-89.

33. Chow, SC, Shao, J, Wang, H, Lokhnygina, Y. Sample size calculations in clinical research, 3rd ed. Boca Raton, Florida: Taylor \& Francis/CRC; 2010.

34. Warren C, Keys J, Pierce-Talsma S. Osteopathic cranial manipulative medicine in the setting of concussion. J Am Osteopath Assoc 2018;118:e41-2.

35. Schwartzberg L, Aslanyan L, Angelo N, Mancini J, Kooyman PS, Abu-Sbaih R, et al. Cranial strain patterns associated with concussions. J Am Osteopath Assoc 2020;120:601-6. 